Franck Guérin Conseil

nouveaun services

aur laboratoires

d'analyses medicales

Aujourd'hui, la réorganisation des structures, le regroupement d'activités et la mise en place de plateaux techniques sont des enjeux importants pour les laboratoires. C'est dans cette perspective que Franck Guérin , ancien collaborateur au sein de la Société SYSMEX propose ses propres services de conseils dans la gestion des laboratoires

\section{Tosoh Bioscience}

Frédéric Bengold a été nommé en juillet 2004 Directeur Général de Tosoh Bioscience France. II succède ainsi à Eric Hermann. Diplômé de Biochimie à Paris VI et titulaire d'un MBA de l'IAE Paris, Frédéric Bengold a travaillé de 1992 à 2002 chez bioMérieux dans des fonctions commerciales et marketing, en France, en Allemagne et à l'International. Avant de rejoindre Tosoh Bioscience France et depuis février 2002, il était

\section{Square}

Square est une société européenne spécialisée dans les projets de construction et d'aménagement "clés en main " des laboratoires d'analyses médicales. L'entreprise propose de nombreux services " à la carte " parmi lesquels :

- Recherche et conseil dans l'implantation du local et/ou de charte graphique

- Réaménagement de l'espace en laboratoire d'analyse (accueil, secrétariat, bureaux, parties techniques)

- Concernant les parties techniques : agencement des paillasses, mobiliers, cloisons après sept années d'exercice dans différents laboratoires hospitaliers et privés et dix dans l'organisation et l'informatisation de ces mêmes laboratoires en France et à l'étranger. Apporter de nouvelles idées d'organisation et de productivité en les adaptant aux besoins des laboratoires, mettre en place une nouvelle organisation liée à un regroupement, à un nouveau système de gestion informatique ou à une évolution de l'activité, telles sont aujourd'hui les compétences

directeur marketing Europe de Sangstat (Groupe Genzyme), société pharmaceutique spécialisée en transplantation. La direction des Ventes est toujours assurée par patrice Bouquet, la direction du Support Clients et de la Communication par Philippe Souquières.

Ensembles, ils souhaitent continuer à apporter aux biologistes français, des solutions innovantes et performantes, dans un souci constant de service et de qualité.

(hématologie, bactériologie, salle noire), etc..

Square élabore les plans du laboratoire en $3 D$ avec film et peut aussi assurer le suivi du chantier sur place. Les différentes étapes du projet comportent l'étude des besoins du client, le sommaire de l'avant-projet, le détail de l'avant-projet, les appels d'offres et enfin la gestion complète et le suivi du chantier. L'espace étant aménagé avec des cloisons amovibles, le projet peut évoluer avec le temps. D'autre part, la société applique le règlement du GBEA au niveau des flux de personnes ainsi que des produits et les que Franck Cuérin met au service des laboratoires.

Pour davantage

d'informations, contactez :

Franck Guérin Conseil

23 rue $A$. Fouilleret

91700 Villiers sur Orge

Tél. : 0870220232

Fax : ol 69514882

Mail : Franck.Cuerinodcileh.com

\section{Tosoh Bioscience \\ 69 rue des sables de sary \\ 45770 Saran \\ Tél. : 0238724444 \\ Fax : $0238724440=$}

réalisations sont contrôlées par la DDASS à la réception du chantier.

En travaillant sur des projets de construction et de rénovation, Square propose ainsi un projet sur mesure qui tient compte des besoins de chaque laboratoire.

\section{Square}

56 rue Georges Clémenceau 06400 Cannes

Tél. : 0492983420

Fax: 0492983429

Mail : infoDespacesquare.com
Accord de distribution entre DPC et Thermo Electron portant sur les lignes de produits: chimie clinique Honelab et TCAutomation

Le 18 juin 2004, les sociétés DPC et Thermo Electron ont signé un accord de distribution concernant les lignes de produits suivantes : systèmes de chimie clinique Thermo Konelab et TCAutomation. DPC devient ainsi le distributeur exclusif des systèmes de chimie clinique Konelab, dans le secteur du diagnostic médical et vétérinaire pour l'Espagne, le Portugal et le Benelux.

En France comme en Allemagne, compte tenu de l'implantation forte de Thermo sur ces deux territoires l'accord prévoit une coopération de distribution. Les objectifs des deux filiales françaises sont complémentaires : DPC souhaite consolider et étendre sa position en immunoanalyse en offrant des solutions mixtes chimie clinique - immunoanalyse robotisées, conçues grâce à la modularité de la gamme TCAutomation.

Thermo désire renforcer l'implantation de ses gammes sur son marché traditionnel. Ensemble DPC et Thermo ont une gamme adaptée à la majorité des segments du marché français.

Cette alliance permet à ses deux sociétés spécialistes de domaines complémentaires de mettre en commun de larges moyens pour le développement de plateformes robotisées consolidant biochimie et immunoanalyse. 